\title{
Mechanismen des Erst- und Zweitspracherwerbs
}

\author{
Mechanisms of First and Second Language Acquisition
}

Autor
Institut
W. Klein

Max-Planck-Institut für Psycholinguistik, Nijmegen (Niederlande)
Schlüsselwörter

Erstspracherwerb

Zweitspracherwerb bei

Kindern und Erwachsenen

Key words

first language acquisition second language acquisition in childhood and adulthood
Bibliografie

DOI 10.1055/S-2007-98581 S

Sprache Stimme Gehö $\mathrm{r}$

2007;31:138-143

(c) Georg Thieme Verlag KG

Stuttgart - New York

ISSN 0342-0477

Korrespondenzadresse

Prof. Dr. W. Klein

Max-Planck-Institut für

Psycholinguistik

P.O. Box 310

NL-6500 AH Nijmege $n$

wolfgang.klein@mpi.n

\section{Zusammenfassung}

Über den Übergang vom angeborenen Sprach vermögen zur tatsächlichen Beherrschung eines oder mehrere r sprachlicher Systeme wissen wir zum einen sehr viel, zum andern aber sehr wenig. Die Fülle der einzelnen Befunde ist schier unüberschaubar; aber sie schließen sich nicht zu einer klaren Vorstellung über die Prinzipien zusammen, die den Spracherwerb bestimmen. Die Gründe dafür liegen darin, dass sich dieser Prozess unter sehr unterschiedlichen Bedingungen vollzieht, dass sich die Fähigkeiten zum Sprachlernen ändern, dass dieser Prozess sehr lange dauert, dass seine Untersuchung insbesondere der Bedeutungsseite methodisch schwierig ist, dass er sehr viele unterschiedliche Fertigkeiten betrifft und schließlich daran, dass unser Verständnis, wie sprachliche Systeme überhaupt funktionieren, noch sehr lückenhaft ist. Dennoch gibt es einzelne übergreifende Befunde, von denen drei diskutiert werden: (1) Vergleicht man Kinder und Erwachsene, so gibt es einen deut lichen Alterseffekt: Während der Erstspracherwerb in der Regel zu perfekter Beherrschung führt, gilt dies für den Zweitspracherwerb Erwachsener fast nie. Allerdings konnte bislang für jede untersuchte sprachliche Eigenschaft gezeigt werden, dass auch Erwachsene sie perfekt lernen können. Sie tun es bloß nicht. (2) Beim Zweitspracherwerb von Kindern spielt das Alter keine wesentliche Rolle . (3) Kinder legen wesentlich mehr Wert auf formale Richtigkeit als Erwach sene - sie sind die besseren „Kopierer" vorhan dener Systeme.

\section{Abstract}

Language acquisition is the transition between the language faculty, with which we are born as a part of our genetic endowment, to the mastery of one or more linguistic systems. There is a plethora of findings about this process; but these findings still do not form a coherent picture of the principles which underlie this pro cess. There are at least six reasons for this situation. First, there is an enormous variability in the conditions under which this process occurs. Second, the learning capacity does not remain constant over time. Third, the process extends over many years and is therefore hard to study. Fourth, especially the investigation of the mea ning side is problem-loaded. Fifth, many skills and types of knowledge must be learned in a more or less synchronised way. And sixth, our understanding of the functioning of linguistic systems is still very limited. Nevertheless, there are a few overarching results, three of which are discussed here: (1) There are salient differences between child and adult learners: While child ren normally end up with perfect mastery of the language to be learned, this is hardly ever the case for adults. On the hand, it could be shown for each linguistic property examined so far, that adults are in principle able to learn it up to perfection. So, adults can learn everything perfectly well, they just don't. (2) Within childhood, age of onset plays no essential role for ultimate attainment. (3) Children care much more for formal correctness than adults - they are just better in mimicking existing systems. It is argued that age does not affect the ,construction capacity" - the capacity to build up linguistic systems - but the „,copying faculty", i.e., the faculty to imitate an existing system. 


\section{Lernziel}

Kenntnisse über Besonderheiten des Erst- und Zweitspracherwerbs sowie des Zweitspracherwerbs bei Kindern und Erwachsenen.

\section{Was ist „Spracherwerb"?}

Das Wort „Sprache" wird, wie die meisten Wörter einer Sprache, in ganz verschiedener Weise verwendet. Seit Ferdinand de Saussure - un d im Grunde schon bei vielen früheren Autoren - unterscheidet man gewöhnlich zwischen drei Begriffen von „Sprache". Zum ersten versteht man darunter das einem jeden Menschen angeborene Sprachvermögen, das Teil unserer genetischen Ausstattung ist und das uns, soweit wir wissen, von allen anderen Arten unterscheidet. Zum zweiten meint man damit das einzelne sprachliche System, wie z.B. Tagalog, Deutsch, Kpelle, Guugu Yimidhirr, Urdu oder Akkadisch. Solche Systeme gibt es derzeit vielleicht 6000-7000; wie viele es wirklich sind, weiß man nicht, denn es gibt zwischen ihnen keine klare Abgrenzung. Man kann die Mundart des Emmentals und das Hochdeutsche ebenso gut als zwei Varianten einer Sprache wie als zwei eigenständige Sprachen werten. Beides sind hochkomplexe Ausdruckssysteme, die viele Ähnlichkeiten, aber auch viele Verschiedenheiten aufweisen. Alle sprachlichen Systeme sin d Produkte des genetischen Sprachvermögens, die sich im Lauf vieler Jahrtausende zu einer bestimmten Form entwickelt haben, aber immer auch eine erhebliche innere Variabilität aufweisen. Niemand wird mit einem solchen System im Kopf geboren; aber allem Anschein nach kann sich jedes Kind jedes beliebige sprachliche System aneignen; dies hängt nur davon ab, in welche soziale Umgebung es geboren wird und wo es aufwächst. Der dritte Begriff von Sprache schließlich ist die Fähigkeit, ein solches System zur Kommunikation mit anderen zu nutzen. Der Begriff „Kommunikation" ist dabei sehr weit gefasst. Eine Sprache dient nicht nur der alltäglichen Verständigung, sondern beispielsweise auch als Informationsspeicher, der es erlaubt, komplexe Wissenssysteme aufzubauen. Sie ist damit eine zentrale Voraussetzung der menschlichen Kognition.

Diese drei Begriffe von Sprache sind zwar klar zu trennen, hängen aber in vielfältiger Weise miteinander zusammen. Ein solcher Zusammenhang ist der „Spracherwerb", also der Übergang vom angeborenen Sprachvermögen zu - nun, wozu eigentlich? Ist es der Übergang vom Sprachvermögen zum einzelnen System, etwa dem Deutschen, oder ist es der Übergang vom Sprachvermögen zu der Fähigkeit, sich mithilfe eines solches Systems zu verständigen? Das ist keineswegs dasselbe. Ein sprachliches System zu erwerben, heißt, Form und Bedeutung von Ausdrücken, einfachen wie zusammengesetzten, zu erlernen. Dieses Wissen ist aber nur ein Teil der viel umfassenderen Fähigkeit, Gedanken, Wünsche und Gefühle in einer Serie von Schritten in eine Schallfolge (oder eine andere wahrnehmbare Ausdrucksform) umzusetzen un d umgekehrt, eine Schallfolge so zu deuten, dass man die ihr zugrunde liegenden Gedanken, Wünsche und Gefühle rekonstruieren kann. Dementsprechend versteht man unter „Spracherwerb" auch die allmähliche Ausbildung der Fähigkeit, kommunikative Aufgaben mit sprachlichen Mitteln zu lösen. Das Verhältnis zwischen diesen beiden Begriffen von Spracherwerb ist verwickelt. Das sieht man besonders deutlich, wenn ein Kind sich mehrere Systeme gleichzeitig aneignet, also bei früher Mehrsprachigkeit.
Wie immer man nun den Spracherwerb versteht, als Erlernen eines Ausdruckssystems oder als die Entwicklung der Fähigkeit, Gedanken in Schallfolgen umzusetzen und umgekehrt - zweierlei ist klar: (a) er ist ein extrem komplexer und langwieriger Prozess, und (b) er ist nicht regellos, sondern folgt bestimmten Mechanismen oder Prinzipien. Und so sollte die Wissenschaft denn auch in der Lage sein, diese Prinzipien anzugeben. Das ist sie aber derzeit nicht - und dafür gibt es mindestens sechs Gründe. Diese Gründe sollen hier nicht nur erläutert werden, um die Spracherwerbsforscher ein wenig zu entschuldigen, sondern auch, um deutlich zu machen, dass allgemeine Aussagen auf diesem Gebiet nur selten mehr als große Sprüche sind und sein können.

\section{Warum ist der Spracherwerb so schwierig zu beschreiben?}

\section{Disparatheit}

Das erste Problem liegt darin, dass der Prozess des Spracherwerbs unter sehr verschiedenen Bedingungen ablaufen kann. Im Titel sind „Erstspracherwerb " und „Zweitspracherwerb " einander gegenüber gestellt. Das ist auch üblich, aber in Wirklichkeit ist es eine krasse Vereinfachung. Es kann ja durchaus sein, dass ein Kind von Anfang an mehrere Sprachen lernt. Das ist sogar sehr häufig so - in der Geschichte der Menschheit und auch in der Gegenwart wahrscheinlich der Normalfall. Man kann sich dies durch zwei Zahlen vergegenwärtigen. In der Welt gibt es, wie schon gesagt, etwa 6000-700 0 Sprachen. In de $\mathrm{UN}$ sind fast 200 Staaten vertreten. Also gibt es - bei massiven Schwankungen im einzelnen - pro Staat ungefähr 35 Sprachen (in Wirklichkeit sind es noch viel mehr, weil ja viele Sprachen in mehr als einem Staat gesprochen werden). Das heißt natürlich nicht, dass jedes Kind im Schnitt 35 Sprachen lernt, aber es heißt, dass ein Kind in aller Regel mit mehreren Sprachen konfrontiert ist und auch mehrere Sprachen lernen muss. Die Aneignung der einzelnen Systeme kann dabei mehr oder minder zeitversetzt sein vom gleichzeitigen Beginn bei Geburt bis zu jenen Fällen, in denen zumindest eine Sprache schon weithin beherrscht wird. Und dies geht gleitend über in den Zweitspracherwerb von Jugendlichen und Erwachsenen.

Der Zeitpunkt, in dem der Zweit-, Dritt-, Viertspracherwerb einsetzt, ist aber nur einer von vielen Faktoren, die zu einer bestimmten Form des Spracherwerbs führen. Ein anderer besonders wichtiger Faktor ist die Art, in der der Lerner Zugang zu dem hat, was er lernen soll. Hier pflegt man zwischen ,ungesteuertem" und ,gesteuertem " Zweitspracherwerb zu unterscheiden. Mit ersterem meint man das Erlernen in der alltäglichen Kommunikation mit Sprechern der zu lernenden Sprache, mit letzterem das Erlernen im Unterricht, also durch gezielte Intervention in einen natürlichen Prozess. Diese Unterscheidung ist jedoch sehr vergröbernd. Zum einen kann es beim „ungesteuerten" Erwerb durchaus auch gezielte Eingriffe geben, z.B. mehr oder minder systematische Korrekturen ; zum andern gibt es höchst unterschiedliche Formen des Sprachunterrichts, vom Rollenspiel in der Fremdsprache, der dem ungesteuerten Erwerb relativ nahe steht, bis zum bloßen Einpauken von Vokabeln und grammatischen Regeln, wie man ihn aus dem ganz traditionellen Unterricht kennt. Anders gesagt, es gibt nicht „den" Spracherwerb, weder bei der ersten noch bei weiteren Sprachen, und deshalb ist es auch schwer möglich, von ,den " Prinzipien des Spracherwerbs zu reden. 


\section{Alterseffekte}

Wir wissen auch nicht, ob das Sprachvermögen bei Geburt bei allen Menschen gleich ist. Man nimmt allgemein an, dass ein jeder in der Lage ist, eine jede beliebige Sprache zu lernen - als Erstsprache. Das ist zwar nicht erwiesen, aber alle Erfahrung spricht dafür. Das schließt jedoch nicht aus, dass es angeborene Unterschiede in der Art und Weise, wie man Sprachen lernt, gibt. Wir wissen es einfach nicht. Was wir jedoch sicher wissen, ist, dass sich das Lernvermögen im Laufe des Lebens erheblich ändert. Das zeigt sich am deutlichsten in der allgemeinen - und auch von der Wissenschaft bestätigten - Erfahrung, dass man als Erwachsener nur selten eine Sprache ,perfekt " lernen kann, während dies beim Erstspracherwerb von Kindern der Normalfall und beim Zweitspracherwerb von Kindern zumindest nicht ungewöhnlich ist.

\section{Dauer}

Bis ein Kind zumindest eine Sprache perfekt beherrscht, dauert sehr lange - zehn Jahre mindestens.

der modernen Linguistik

wird oft vom ,schnellen und mühelosen Spracherwerb des Kindes auf der Grundlage eines unzulänglichen Inputs" geredet - ein zentrales Argument für die bekannte Chomskysche Vorstellung, dass ein großer Teil der Grammatik angeboren ist und daher nicht gelernt zu werden braucht. Aber es gibt viele Belege dafür, dass auch Kinder im fortgeschrittenen Schulalter Probleme mit bestimmten grammatischen Regeln haben, vom Wortschatzerwerb oder der Fähigkeit, eineen komplexen Text zu konstruieren, ganz zu schweigen. Einen Prozess zu erforschen, der sich im Regelfall über ein gutes Jahrzehnt erstreckt, erfordert einen außerordentlichen Aufwand, der Finanzvolumen und Förderdauer eines nor malen DFG-Projektes bei weitem übersteigt.

\section{Methodische Probleme}

Das sprachliche Wissen kann man nicht sehen. Sehen oder hören kann man nur die ,äußere Form" der Sprache, also Lautfolgen, Zeichen auf dem Papier oder Gebärden. Nicht diese jedoch machen ein sprachliches System aus, sonderrn ihre regelhafte Verbindung mit bestimmten Bedeutungen. Dieses Wissen ist irgendwo im Kopf gespeichert und dort wird es zur Sprachproduktion oder zum Sprachverstehen abgerufen. Wenn man das sprachliche Wissen eines Lerners zu einem gegebenen Zeitpunkt ermitteln will, genügt es nicht festzustellen, was er sagt, sondern man muss auch feststellen, was er sagen will; ebenso genügt es nicht festzustellen, was er hört oder sieht, sondern wie er das Gehörte oder Gesehene deutet. Das ist sehr schwierig und aufwendig: wie ,misst" man, welche Vorstellungen ein Kind mit der Lautfolge/nox/verbindet? Hier hat auch die Hirnforschung, so spektakulär sich die modernen bildgebenden Verfahren ausnehmen, bislang keine nennenswerten Fortschritte gebracht. Zwar kann man mit diesen Verfahren in gewissen Grenzen Änderungen des Blutflusses oder der elektrischen Aktivität in bestimmten Arealen messen; aber das sagt uns allenfalls, wo ungefähr sprachliches Wissen verarbeitet wird, nicht aber, wie es zu einem gegebenen Zeitpunkt aussieht und wie es sich entwickelt. Kein buntes Bild eines Hirnareals gibt uns Auskunft, was ein Kind unter/nox/versteht, schon gar nicht, ob es darunter etwas anderes versteht als ein Erwachsener.

\section{Vielfalt des sprachlichen Wissens}

Die Beherrschung einer Sprache umfasst sehr viele einzelne Kenntnisse und Fertigkeiten, die in aller Regel eng miteinander verzahnt sind. Dazu zählen beispielsweise
- die Phonologic d.h. die Aussprache der einzelnen Laute und - eine sehr wichtige, aber schwer zu lernende und so gut wie nie gelehrte Eigenschaft - die Intonation

- die Syntax , d.h. Eigenschaften wie Wortstellung, Rektion, Aufbau von Satzteilen usw.

- die Morphologie, d.h. Konjugation, Deklination, Wortbildung

- das Lexikon, d. h. die einfachen Wörter, aber auch alle zusammengesetzten Ausdrücke, insoweit sich ihre Bedeutung nicht aus der Bedeutung ihrer Teile ergibt (Kollokationen, Phraseologismen usw.)

- Bildung und Verstehen komplexeer Texte, wie etwa Wegbeschreibungen, Instruktionen, Gebrauchsanweisungen - angemessenes interaktives Verhalten, z.B. Rederecht oder Anredeformen, um nur einige zu nennen.

Auch wenn sich der Erwerb dieser Fertigkeiten nicht nach dem Motto „Kraut und Rüben", sondern nach bestimmten Regeln vollzieht, so ist sofort klar, dass es nicht einen einfachen Satz von „Prinzipien des Spracherwerbs " geben kann. Dazu sind diese Fertigkeiten viel zu unterschiedlich; nicht zuletzt sind sie in unterschiedlicher Weise mit anderen Entwicklungsvorgängen etwa der kognitiven und der sozialen Entwicklung - gekoppelt.

\section{Beschränktheit unseres Wissens über Sprachen}

Das letzte Problem, das sich dem Spracherwerbsforscher in den Weg stellt, ist die Unzulänglichkeit unseres Wissens über sprachliche Systeme. Als Linguist gibt man dies ungern zu, aber es ist leider so. Wie viele der rund 6500 Sprachen der Welt sind wirklich gut erforscht? Nehmen wir an, eine Sprache ist, ,gut erforscht", wenn es mindestens drei Grammatiken und drei Wörterbücher gibt - wahrlich ein bescheidenes Kriterium -, dann sind vielleicht 100 Sprachen gut erforscht, also weniger als $2 \%$. Und wenn man nun einmal eine der besterforschten Sprachen der Welt, das Deutsche, betrachtet, so hat kein Linguist bislang eine befriedigende semantische Analyse eines so gängigen Wortes wie „noch" - von Kindern übrigens seh r früh gelernt vorlegen können. Wieso kann man beispielsweise sagen „Goethe war noch krank" un d auch „Noch war Goethe krank", während „Noch Goethe war krank" zumindest sehr merkwürdig ist? Angesichts all dieser Schwierigkeiten beginnt man sich vielleicht zu wundern, dass überhaupt irgendwelche Lehrbücher zum Erst- oder Zweitspracherwerb erscheinen [1, 2]. Aber das Problem ist eigentlich nicht, dass man nichts wüsste. Die Forschung insbesondere der letzten drei Jahrzehnte hat sehr viel an Erkenntnissen zutage gebracht, und dies zu allen Bereichen sprachlichen Wissens, wie sie unter Punkt E genannt wurden. Es ist nur so, dass diese vielen Einzelbefunde kein geschlossenes Bild ergeben. Fast möchte man sagen: Je mehr man weiß, desto weniger kann man sagen. Dies gilt insbesondere für die Frage, ob Erstspracherwerb und Zweitspracherwerb unterschiedlichen Prinzipien folgen. Immerhin - einige allgemeine Aussagen kann man schon treffen, und im Folgenden soll auf drei Beispiele dafür eingegangen werden.

\section{Drei übergreifende Befunde}

\section{Erstsprache bei Kindern - Zweitsprache bei Erwachsenen}

Die meiste $n$ Unterschiede zwischen Erst- und Zweitspracherwerb kann man an drei Faktoren festmachen:

- dem Lebensalter; die Erstsprache lernt man als Kind, weitere Sprachen kann man auch später im Leben lernen; 
- dem sprachlichen Vorwissen, d. h. dem Ausmaß, zu dem man über Kenntnisse anderer Sprachen als der gerade zu lernenden verfügt;

- dem Zugang, d.h. der Art und Weise, in der die zu lernende Sprache vermittelt wird - in der alltäglichen Kommunikation, im Unterricht, in einer Verbindung beider.

Alle drei Faktoren sind offensichtlich graduell. Vereinfachen wir die Sache aber einmal auf den Gegensatz zwischen dem monolingualen Erstspracherwerb von Kindern und dem Zweitspracherwerb von Erwachsenen (wobei der Unterschied gesteuert ungesteuert für das Folgende keine Rolle spielt). Dann zeigt die bisherige Forschung zweierlei [3]:

A. Der Erstspracherwerb von Kindern führt fast immer zu perfekter Beherrschung, der Zweitspracherwerb führt fast nie zu perfekter Beherrschung (manche sagen sogar ,nie", aber das trifft nicht zu). „Perfekt" heißt dabei nicht, wie Goethe reden oder schreiben zu können, sondern, dass die Betreffenden von muttersprachlichen Sprechern als solche angesehen werden.

B. Für jede bislang untersuchte Eigenschaft, von der Aussprache über die Morphologie zu Lexikon und Syntax, konnte gezeigt werden, dass man sie auch noch als Erwachsener perfekt lernen kann (wobei mit „Erwachsenen" zumeist Studenten gemeint sind, denn Untersuchungen über den Spracherwerb in fortgeschrittenem Lebensalter gibt es so gut wie nicht).

Das Fazit ist also:

C. Erwachsene können eine zweite Sprache perfekt lernen, tun es aber nicht.

Dies wirft ein bemerkenswertes Licht auf die möglichen Ursa chen des Alterseffekts. Früher hatte man oft angenommen, dass es für den Spracherwerb eine „Prägephase" gibt, wie man sie für viele biologische Reifungsprozesse kennt . Nur in diesem Zeit fenster verfügt das Gehirn über die erforderliche Plastizität. Diese Vorstellung einer solchen ,critical period " ist sicher nicht richtig; es gibt auch kaum entsprechende Befunde aus der Hirnforschung. Das schließt nicht aus, dass biologische Verände rungen über die Lebensspanne eine Rolle spielen; abe r sie sind komplexer, und es sind nicht die einzigen Faktoren.

\section{Zweitsprache bei Kindern verschiedenen Alters}

Ein Kind kann eine zweite - vielleicht gar eine dritte und vierte - Sprache von Geburt an, aber auch zeitversetzt lernen. Wie wirkt sich das Lebensalter zu Beginn des Erwerbs aus? Gilt hier die Regel ,je früher, desto besser" - eine Regel, die beispielsweise für einen Fremdsprachunterricht im Kindergarten spräche ? Oder gilt umgekehrt die Rege 1 ,je älter, desto besser", weil der Erwerb einer zweiten Sprache erst einsetzen sollte, wenn die Kenntnisse in der Erstsprache einigermaßen gefestigt sind und so wechselseitige Störungen vermieden werden?

Wie so oft, gleicht der Forschungsstand - jedenfalls aus der Ferne betrachtet - eher einer Nebelwolke, weil es ganz divergierende Befunde gibt [4]. Aber das Fazit in entsprechender Vereinfachung ist:

D. Das Alter spielt beim Zweitspracherwerb von Kindern kaum eine Rolle.

Dies heißt nicht, dass es keine Rolle spielt. Aber in der Kindheit kann man eine zweite Sprache perfekt lernen, ganz gleich wann man damit anfängt. $\mathrm{Ob}$ und wie dies gelingt, hängt von anderen Faktoren ab, beispielsweise der Intensität, mit der man sich der Aufgabe widmet, oder der Motivation. Man beachte, dass es hier um das Endergebnis geht; ob der Verlauf des Erwerbsprozesses immer gleich ist, ist eine andere Frage. Aber auch bei Kindern gleichen Alters ist der Verlauf des Erwerbsprozesses oft deutlich verschieden.

\section{Erst- und Zweitsprache bei Kindern - Zweitsprache bei Erwachsenen}

Das Lebensalter zeitigt Wirkungen; sie sind aber, wie wir gesehen haben, nicht auf einen einfachen Nenner zu bringen. Zu den unter A-D genannten allgemeinen Befunden kommt ein wei terer hinzu:

E. Das Alter wirkt sich bei den einzelnen sprachlichen Eigen schaften unterschiedlich aus. Die stärksten Effekte zeigen sich in Phonologie und Morphosyntax, und hier wiederum in der Flexionsmorphologie. Hingegen sind Wortschatzerwerb oder eher pragmatische Fähigkeiten weniger betroffen.

Erwachsene Zweitsprachlerner, die nicht unter dem Einfluss eines bestimmten Unterrichts stehen, bilden durchweg eine besondere Sprachform - eine „basi c variety" [5] - aus. Die Sätze dieser Basisvarietät folgen einigen ganz einfachen Regeln - beispielsweise der Regel ,Jenes Argument, das den höchsten Grad an Kontrolle über die Situation ausübt, steht zuerst, kurz: Agens zuerst" oder „Alte vor neuer Information". Das auffälligste Merkmal dieser Sprachform ist das fast völlige Fehlen einer Flexion genauer gesagt, einer funktionalen Flexion. Zwar tauchen Wörter oft in etwas unterschiedlichen Formen auf, aber diese Variation scheint keinem Zweck zu dienen. Bei Kindern ist dies völlig anders, und zwar ganz gleich, ob es sich um die Erstsprache oder die Zweitsprache handelt. Sie zeigen ein ausgesprochenes Sensorium für Flexionsmorphologie ; sie machen es nicht gleich richtig, aber sie achten sehr darauf, und sie beherrschen die entsprechenden Regelhaftigkeiten zu einem sehr frühen Stadium des Erwerbsprozesses.

Dies ist einer der auffälligsten Unterschiede zwischen Kindern und Erwachsenen im Spracherwerb . Ist er rein altersbedingt, vielleicht gar durch einen Reifungsprozess im Gehirn, in dessen Gefolge der Sinn für die Flexion verloren geht? Das kann nicht sein, denn Erwachsene, di e ein e zweite Sprache im Unterricht erlernen, entwickeln keine „Basisvarietät", sondern sie eignen sich durchaus ein gewisses Maß an Flexionsmorphologie an. Alles andere wäre auch enttäuschend, wenn man daran denkt, welches Gewicht den oft verwirrenden Regeln von Deklination und Konjugation in der Schule beigemessen wird; wir erinnern uns mit Grauen des Lateinunterrichts. Diese starke Betonung der Flexion spiegelt sicher nicht die ,natürlichen Erwerbsmechanismen" des Zweitspracherwerbs wider, genauer gesagt, des Zweitspracherwerbs Erwachsener, die sich um die Flexion nicht groß scheren. Aber das Ergebnis des Schulunterrichts zeigt, dass Erwachsene die Fähigkeit nicht grundsätzlich verloren haben. Sie machen nur, auf sich gestellt, wenig Gebrauch davon.

Woran also liegt der Unterschied zwischen Kindern und Erwachsenen? Eine wirklich schlüssige Antwort gibt es nicht. Aber man erhält doch gewisse Hinweise, wenn man mit der Linse ein wenig näher heranfährt und den Erwerb verschiedener Flexionsregeln miteinander vergleicht.

\section{Nastja und Dascha}

Im Folgenden wird auf einige Befunde aus einem Forschungsprojekt eingegangen, das von Christine Dimroth (MP I für Psycholinguistik, Nijmegen) und Ursula Stephany (Universität Köln) durchgeführt wurde. Es ist - wie so viele Untersuchungen auf 
diesem Gebiet - eine Fallstudie, in der über anderthalb Jahre untersucht wurde, wie zwei russische Kinder, Nastja und Dascha, Deutsch lernen . Beide konnten bei der Ankunft kein Deutsch, beide gingen von Anfang an zur Schule, beide wurden zwar auf Deutsch, aber nicht im Deutschen unterrichtet, beide sind Geschwister, d.h. die sozialen und familiären Bedingungen sind annähernd gleich. Der Unterschied liegt darin, dass Nastja bei Ankunft etwa acht und Dascha etwa 14 Jahre alt war; ein Unterschied von sechs Jahren wiegt in diesem Lebensalter viel. Ich greife aus den Ergebnissen einige aus dem Bereich der Flexionsmorphologie heraus, die für unser Thema wichtig sind (die Darstellung ist sehr vereinfacht, Einzelheiten finden sich in [1] und der dort zitierten Literatur):

A. Subjekt-Verb-Kongruenz. Im Deutschen kongruiert das finite Verb mit dem Subjekt. Diese Markierung hat einen gewissen funktionale $n$ Wert, weil sie oft das einzige Mittel ist, zwischen Subjekt und Objekt zu unterscheiden : Die Mutter betreuen die Kinder - Die Mutter betreut die Kinder. Diese Kongruenz wird von beiden Kindern rasch und offenbar ohne große Problem e gelernt. Nennenswerte Unterschiede gibt es nicht, einmal abgesehen davon, dass das jüngere Kind etwas schneller ist.

B. Vergangenheitsmarkierung. Das Deutsche hat zwei Vergangenheitsformen, Präteritum und Perfekt (neben dem ehe rr seltenen Plusquamperfekt). In der gesprochenen Sprache überwiegt das Perfekt, aber beide Kinder lesen auch Märchen und sonstige Texte, in dene $n$ das Präteritu $m$ durchaus nich $t$ selten ist. Wie lernen sie die Tempusmarkierung? Im Großen und Ganzen in vier sich teilweise überlappenden Schritten:

(1) Zuerst wird durchweg die Präsensform verwendet, auch zur Bezeichnung der Vergangenheit. (2) Anschließend findet sich bei beide $n$ Kindern oft ein - $t$ zur Vergangenheitsmarkierung; woher dieses Suffix kommt, ist nicht ganz klar - entweder aus dem regelmäßigen Präteritum (lach-te) oder aus dem Partizip (ge-lach-t). (3) Danach wird das Perfekt gelernt. (4) Zuletzt tauchen die Präteritalformen auf.

Dieser Ablauf ist bei beiden Kindern annähernd gleich. Es gibt nur einen wesentlichen Unterschied: das jüngere Kind be achtet - zunächst noch mit Fehlern - von Anfang an den Unterschied zwischen ,haben-Perfekt " und ,sein-Perfekt" ; das ältere bildet das Perfekt immer mit haben und bleibt bis zum Ende der Beobachtungszeit dabei. Man beachte, dass haben und sein beim Perfekt dasselbe leisten; nur muss man je nach Verb manchmal das eine, manchmal das andere wählen.

Sonst ist es zwar genauso verständlich, aber halt nicht „richtig".

C. Pluralmarkierung. Nur wenige Sprachen haben so viele Pluralformen wie das Deutsche : Ente - Enten, Vater - Väter, Lamm - Lämmer, Kuchen - Kuchen, Kuh - Kühe, Tag - Tage, Auto - Autos, um nur die wichtigsten zu nennen. Einen vernünftigen Grund hat diese Variation nicht - es ist ein zweifelhaftes Geschenk unserer Vorfahren . Wie gehen die beiden Kinder mit dieser Vielfalt um? Hier zeigen sich deutliche Unterschiede. Das jüngere Kind variiert von Anfang an stark, trifft manchmal die richtige Form, manchmal nicht, macht aber im Laufe der Zeit alle deutschen Ungereimtheiten richtig nach; bei neuen Wörtern wird oft die Singularform beibehalten. Das ältere Kind markiert den Plural von Anfang an mit -(e)n und bleibt weitgehend dabei. Das ist eigentlich eine einfache und klare Lösung - nur nicht die, die das Deutsche gewählt hat.
D. Adjektivflexion. Ist die deutsche Pluralmarkierung schon ein wenig aufwändig, so is t die Adjektivflexion fast bizarr: kaltes Bier, das kalte Bier, mit kaltem Bier, mit einem kalten Bier . Einen funktionalen Wert hat diese Markierung kaum, und so haben andere germanisch e Sprachen sie denn auch weithin abgeschafft oder zumindest vereinfacht. Wie gehen di e beiden russischen Kinder damit um - wobei man beachten muss, dass das Russische gleichfalls eine stark ausgebildete Nominalflexion hat? Nastja lernt sie, Dascha lernt sie nicht - sie verwendet zu Beginn die unflektierte Form (kalt) und wechselt dann zu mehr oder minder konstantem -e (kalte).

Wie immer be i Corpusstudien sind die Ergebnisse etwas differenzierter als hierdargestellt. Aber es zeichnet sich ein klares Bild ab, das man auf folgendes Schlagwort bringen kann:

Das jüngere Kind geht auf formale Richtigkeit und opfert zur Not die Funktion (z.B. Pluralmarkierung), das ältere Kind opfert die formale Richtigkeit (z.B . Unterschied zwischen haben-Perfekt und sein-Perfekt, richtige Pluralformen, Adjektivflexion ) und versucht, bestimmte funktionale Unterschiede zu wahren. Der Gegensatz ist nicht absolut, ebenso wie der Gegensatz zwischen „,nicht-funktional" und „funktional" kein absoluter ist.

Diese gegenläufige Entwicklung zwischen dem Spracherwerb von Kindern und dem Zweitspracherwerb von Erwachsenen ist eine Tendenz: die Präferenzen verschieben sich. Es ist nicht so, dass Erwachsene bestimmte Eigenschaften nicht mehr lernen könnten. Sie legen bloß weniger Wert darauf als Kinder. Dies schließt nicht aus, das s sich das Lernvermögen selbst nicht ändert. Einer der Gründe für das ,Altersparadox" könnte ja durchaus sein, dass manches zwar nach wie vor möglich ist, aber sehr viel mehr Mühe macht . Darauf soll im letzten Abschnitt kurz eingegangen werden.

\section{Konstruktionsvermögen - Kopiervermöge n - Kommunikationsvermögen}

In der sprachwissenschaftlichen Tradition wird das angeborene Sprachvermögen als etwas relativ Monolithisches gesehen. Das gilt ganz besonders, aber keineswegs nur für die Spracherwerbsforschung im Rahmen der generativen Grammatik. In Wirklichkeit ist e s aber eine komplexe Ansammlung von angeborenen, teils speziesspezifischen, teils auch bei anderen Arten zu findenden Fähigkeiten, deren Zusammenwirken den Aufbau und das Erlernen sprachlicher Systeme möglich macht. Jede einzelne dieser Fähigkeiten - Hör - und Artikulationsvermögen, hoch strukturiertes und schier unerschöpfliches Gedächtnis, das Vermögen, Assoziationen zu bilden, Schlüsse zu ziehen und was immer - ist in vielfältiger Weise variabel. Diese Variabilität besteht zwischen Individuen; si e zeigt sich auch beim selben Individuum über die Lebensspanne hinweg. Es ist nicht das Sprachvermögen insgesamt, das sich ändert, es sind seine einzelnen Komponenten und das Zusammenspiel dieser Komponenten . Dies kann man nach Belieben herunterbrechen, bis auf die Fähigkeit, die Grundfrequenz im Schallsignal zu erkennen, im Gegensatz zu der Fähigkeit, Formanten im Schallsignal zu erken nen. Und in der Tat könnten ja beide Fähigkeiten unabhängig voneinander gestört sein.

Die einzelnen Fähigkeiten stehen nun allerdings nicht unver bunden nebeneinander, sie wirken zusammen. Es gibt, so scheint mir, drei große, klar zu trennende Formen des Zusammenwirkens. Dies ist zu m einen das Vermögen, sprachliche Systeme aufzubauen - das heißt, Ausdrucksträger mit Bedeutungen zu assoziieren und aus einfachen Ausdrücken (,Wörtern" ) kom plexe Ausdrücke (,flektierte Formen, Satzteile, Sätze, Texte") zu 
bilden. Als unsere Vorfahren vor langen Jahren die ersten linguistischen Systeme konstruiert haben, mussten sie bereits über dieses Konstruktionsvermögen verfügt haben . Ebenso müssen sie die Fähigkeit besessen haben, mit ihresgleichen zu kommunizieren; man kann sich nicht vorstellen, dass ein besonders talentierter Neandertaler sich die erste Sprache heimlich ausge dacht und dann seiner Familie und seinen engsten Freunden mitgeteilt hat (wie denn?) . Dieses Kommunikationsvermögen ist gleichfalls Teil unserer genetischen Ausstattung, und zwar unabhängig vom Vorhandensein eines sprachlichen Systems ; sobald es ein solches System zur Verfügung hat, erlebt es schier eine Explosion an Ausdrucksmöglichkeiten, die anderen Arten nicht zur Verfügung stehen. Davon ganz zu trennen ist nun das Vermögen, ein bereits vorhandenes System zu kopieren. Wenn jemand lispelt, wenn jemand Deutsch mit englischem Akzent redet, wenn jemand sagt ,Ich gestern helfen dir" statt „Ich habe dir gestern geholfen", so hat er ein sprachliches System ausgebildet, das so auc $h$ von einer große $n$ Sprachgemeinschaf $t$ ge sprochen werden könnte. Es ist nicht minder eine Sprache als das Deutsche, es ist nur anders, als die Deutschen normalerweise sprechen. Ein solche $r$ Lerne $r$ verfügt nach wie vo $r$ über das Konstruktionsvermögen, nur sein Kopiervermögen hat gelitten: er macht es, aber er macht es nicht genau so wie andere. In den unterschiedlichen Formen des Spracherwerbs - Erstspracherwerb, Zweitspracherwerb, mit allen möglichen Zwischenstufen - entwickeln sich diese drei Fähigkeiten in unterschiedlicher Weise. Das Konstruktionsvermögen bleibt zumindest bis lange ins Erwachsenenalter erhalten - wobei individuelle Schwankungen nicht ausgeschlossen sind . Das Kommunikationsvermögen, gleichfalls genetisch gegeben, aber zunächst sehr beschränkt, entwickelt sich massiv mit dem Erwerb eines neuen Systems, gleich ob es das erste oder das zweite ist; dabei legen erwachsene Lerner auf diese Entfaltung besonderes Gewicht . Das Kopiervermögen wird hingegen abgebaut; dabei ist offen, ob dies seinen Grund eher in biologischen Veränderungen - sei es im Gehirn oder den peripheren Sprechwerkzeugen - oder in eine r unterschiedlichen Motivation hat. Kinder müssen sich eine soziale Identität innerhalb einer Gemeinschaft schaffen, und das kann man nur, wenn man genauso spricht, wie es in dieser Gemeinschaft üblich ist. Erwachsene haben eine soziale Identität, und sich eine weitere Identität anzueignen, ist weniger wichtig, mag sogar ihre erste bedrohen. Sie eignen sich daher ein neues sprachliches System an, weil es ihre kommunikativen Möglichkeiten erweitert. Aber sie müssen nicht genauso sprechen wie die muttersprachlichen Sprecher, und sie tun es in der Regel auch nicht.

\section{Fragen zur Selbstkontrolle}

1. Besonderheiten des Erst- und Zweitspracherwerbs

a) Erst- und Zweitspracherwerb verlaufen nach völlig unterschiedlichen Gesetzmäßigkeiten .

b)Diegrundlegenden Prinzipien des Erst- und Zweitspracherwerbs sind vergleichbar.

c) Ob Erst - un d Zweitspracherwerb nach gleichen oder unterschiedlichen Gesetzmäßigkeiten verläuft , hängt wesentlich vom Alter des Lerners ab.

2. Erfolg des Zweitspracherwerbs im Erwachsenenalter

a) Im Erwachsenenalter ist ein perfektes Erlernen einer neuen Sprache nicht mehr möglich. b) Jede de r einzelnen Sprachdimensionen kann auch im Erwachsenenalter noch perfekt erlernt werden, nicht jedoch das gesamte System.

c) Auch Erwachsene können eine neue Sprache noch perfekt erlernen, auch wenn die meisten nicht so weiy kommen.

3. Optimaler Zeitpunkt für das Erlernen einer Zweitsprache

a) Am besten lernt ein Kind eine zweite Sprache zusammen mit seiner Muttersprache, denn je früher, um so besser.

b) Der Zweitspracherwerb sollte erst beginnen, nachde m die Muttersprache ausreichend gefestigt ist.

c) Im Kindesalter gibt es weder eine kritische Periode für den Spracherwerb noch einen optimalen Zeitpunkt für den Beginn des Zweitspracherwerbs. Während der gesamten Kindheit kann eine weitere Sprache perfekt erlernt werden.

\section{Literatur}

1 Ellis $R$. The Study of Second Language Acquisition. Oxford: Oxford University Press. 1994

2 Klann-Delius G. Spracherwerb. Stuttgart: Metzler 1999

3 Birdsong D. (Hrsg). Second language acquisition and the Critical Period Hypothesis Mahwah. NJ: Erlbaum. 1999

4 Singleton D, Ryan L. Language Acquisition: the Age Faktor. Cleevedon: Multilingual Matters LTD, 2004

5 Klein W, Perdue C. The Basic Variety, or: Couldn't natural languages be much simpler? Second Language Research 1997; 13: 301-347

6 Dimroth $C$. Zweitspracherwerb bei Kindem und Erwachsenen - Gemeinsamkeiten und Unterschiede. In: Anstatt T (Hrsg). Mehrsprachigkeit bei Kindern und Erwachsenen. Tübingen: Narr-Francke (im Druck)

\section{Buchbesprechung}

\section{Taschenführer zur ICD-10-Klassifikation psychischer Störungen - Mit Glossar und Diagnostischen Kriterien ICD-10:DCR-10}

Weltgesundheitsorganisation/Horst Dilling et al. (Hrsg.) Übersetzt und herausgegeben von $\mathrm{H}$. Dilling und $\mathrm{H}$. J. Freyberger nach dem englischsprachigen Pocket Guide von J. E. Cooper. 3., vollst. Überarb. u. erw. Aufl. 2006, unter Berücksichtigung der German Modification (GM) der ICD-10, Verlag Hans Huber, Bern, 528 Seiten, Gb, Euro 29,95

Für die nun vorliegende 3 . Auflage de s Taschenführers fan d eine vollständig e Überarbeitung und Ergänzung entsprechend der Firmen-Modification s (GM ) statt . Der Taschen führer enthält die diagnostischen Kriterien für die einzelnen Störungen samt einem Kommentar . Hierbei werden nach einem kurzen Einführungsabschnitt zu jeder Störung die für die Diagnose relevanten Kriterie n aufgeführt. Weiterhin fin den sich Hinweise zur Differential - und Ausschlussdiagnos tik. Hervorzuheben ist insbesondere die kompakte Definition und Beschreibung der einzelnen Störungen, die das Buch für den klinischen Alltag sehr empfehlenswert machen.

M. Ptok, Hannover 